\title{
On the flux of pseudo-Anosov homeomorphisms
}

\author{
VINCENT COLIN \\ KO HONDA \\ FRANÇOIS LAUDENBACH
}

\begin{abstract}
We exhibit a pseudo-Anosov homeomorphism of a surface $S$ which acts trivially on $H_{1}(S ; \mathbb{Z})$ and whose flux is nonzero.
\end{abstract}

57M50; 53C15

\section{Introduction}

Let $S$ be a compact oriented surface with nonempty boundary, $\omega$ be an area form on $S$ and $h$ be an area-preserving diffeomorphism of $(S, \omega)$. Consider the mapping torus $\Sigma(S, h)$ of $(S, h)$, which we define as $(S \times[0,1]) /(x, 1) \sim(h(x), 0)$. Here $(x, t)$ are coordinates on $S \times[0,1]$. If there is a contact form $\alpha$ on $\Sigma(S, h)$ for which $\left.d \alpha\right|_{S \times\{0\}}=\omega$ and the corresponding Reeb vector field $R_{\alpha}$ is directed by $\partial_{t}$, then we say $h$ is the first return map of $R_{\alpha}$. In this note we investigate the following question:

Question 1.1 What is the difference between an area-preserving diffeomorphism $h$ of a surface $(S, \omega)$ and the first return map of a Reeb flow $R_{\alpha}$, defined on $\Sigma(S, h)$ ?

One easily computes that the first return map of $R_{\alpha}$ is $\omega$-area-preserving (cf Lemma 2.1). Question 1.1 can then be rephrased as follows:

Question 1.2 Can every area-preserving $h$ be expressed as the first return map of a Reeb flow $R_{\alpha}$ ?

We emphasize that we are interested in the rigid problem of realizing a given diffeomorphism $h$, instead of its realization up to isotopy. This question is of particular importance when one tries to compute the contact homology of a contact structure adapted to an open book decomposition; see Colin and Honda [3]. The periodic orbits of an adapted Reeb flow that are away from the binding of the open book correspond to periodic points of the first return map. Hence we would like to understand which monodromy maps can be realized by first return maps of Reeb flows. 
It turns out that the answer to Question 1.2 is negative. There is an invariant of an area-preserving diffeomorphism $h$, called the $f l u x$, which is an obstruction to $h$ being the first return map of a Reeb flow. In Section 2 we define the flux and also show that it is easy to modify the flux of a diffeomorphism within its isotopy class.

The case of particular interest to us is when $h$ is pseudo-Anosov. Recall that a homeomorphism $h: S \stackrel{\sim}{\longrightarrow} S$ is pseudo-Anosov if there exist $\lambda>1$ and two transverse singular measured foliations - the stable measured foliation $\left(\mathcal{F}^{s}, \mu^{s}\right)$ and the unstable measured foliation $\left(\mathcal{F}^{u}, \mu^{u}\right)$-such that $h\left(\mathcal{F}^{s}, \mu^{s}\right)=\left(\mathcal{F}^{s},(1 / \lambda) \mu^{s}\right)$ and $h\left(\mathcal{F}^{u}, \mu^{u}\right)=\left(\mathcal{F}^{u}, \lambda \mu^{u}\right)$. The homeomorphism $h$ is a diffeomorphism away from the singular points of the measured foliations. A pseudo-Anosov representative $h$ of a mapping class is unique in the sense that any two pseudo-Anosov homeomorphisms $h_{1}, h_{2}$ in the same mapping class are conjugate via an everywhere smooth diffeomorphism $\phi$ which is isotopic to the identity. In particular, such a $\phi$ sends the stable foliation of $h_{1}$ to the stable foliation of $h_{2}$ and the unstable foliation of $h_{1}$ to the unstable foliation of $h_{2}$. (See Fathi, Laudenbach and Poénaru [5, Exposé 12, Théorème III and Lemma 16 for smoothness].) We define the area form $\omega$ to be given by the product of $\mu^{s}$ and $\mu^{u}$. The form $\omega$ is the unique $h$-invariant area form up to a constant multiple and is singular in the sense that it vanishes at the singular points of the invariant foliations. Now, the pseudo-Anosov case is of special interest since the pseudo-Anosov homeomorphism is a rigid representative in its mapping class (hence the flux can be seen as an invariant of the mapping class) and also since it is known that every contact structure is carried by an open book decomposition whose monodromy is isotopic to a pseudo-Anosov homeomorphism by Colin and Honda [4]. Hence we ask the following question:

Question 1.3 Can every pseudo-Anosov homeomorphism $h$ be expressed as the first return map of a Reeb flow $R_{\alpha}$ ?

The main theorem of this paper is Theorem 2.4, which states that the answer to this question is also negative, ie, the flux is not always zero for pseudo-Anosov homeomorphisms.

\section{The flux}

The goal of this section is to give basic properties of the flux; see Calabi [1]. The discussion will be done more generally on a compact symplectic manifold, since it might be more transparent in that context. 


\subsection{Flux}

Let $(S, \omega)$ be a compact symplectic manifold and $h$ be a symplectomorphism of $(S, \omega)$. Let $h_{*}: H_{1}(S ; \mathbb{Z}) \rightarrow H_{1}(S ; \mathbb{Z})$ be the map on homology induced from $h$ and let $K$ be the kernel of $h_{*}-$ id. Also let $\Gamma$ be a lattice of $\mathbb{R}$ generated by $\int_{\Sigma} \omega$, where $[\Sigma]$ ranges over $H_{2}(S ; \mathbb{Z})$. Then define the map

$$
F_{h}: K \rightarrow \mathbb{R} / \Gamma
$$

as follows: Let $[\gamma] \in K$. Since $\gamma$ is homologous to $h(\gamma)$, one can find an oriented singular cobordism $C$ (mapped into $S$ ) whose boundary consists of $h(\gamma)-\gamma$. We then define

$$
F_{h}(\gamma)=\int_{C} \omega
$$

Two cobordisms $C, C^{\prime}$ with the same boundary differ by an element of $H_{2}(S ; \mathbb{Z})$; hence the quantity is well-defined only up to $\Gamma$. It is straightforward to verify that $F_{h}(\gamma)$ also only depends on the homology class of $\gamma$. The number $F_{h}([\gamma]) \in \mathbb{R} / \Gamma$ is thus well-defined and is called the flux of $h$ along $\gamma$. We say the flux of $h$ is nonzero if the image of $K$ is not $[0] \in \mathbb{R} / \Gamma$.

If $h_{1}, h_{2}$ are two symplectomorphisms of $(S, \omega)$ and $[\gamma]=\left[h_{1}(\gamma)\right]=\left[h_{2}(\gamma)\right]$, then

$$
F_{h_{2} \circ h_{1}}([\gamma])=F_{h_{2}}([\gamma])+F_{h_{1}}([\gamma]) \text {. }
$$

In other words, the flux is a homomorphism, when viewed as a map from the group $\operatorname{Symp}_{0}(S, \omega)$ of symplectomorphisms which act trivially on $H_{1}(S ; \mathbb{Z})$ to the group $\operatorname{Hom}\left(H_{1}(S ; \mathbb{Z}), \mathbb{R} / \Gamma\right)=H^{1}(S ; \mathbb{R} / \Gamma)$. We can also easily modify the flux of any $h \in \operatorname{Symp}_{0}(S, \omega)$ by composing with time-1 maps of locally Hamiltonian flows.

If in addition $\omega=d \beta$, then the form $h^{*} \beta-\beta$ is a closed 1 -form and the flux of $h$ along $\gamma$ can be rewritten as

$$
F_{h}([\gamma])=\int_{\gamma} h^{*} \beta-\beta,
$$

by the use of Stokes' formula. The flux of $h$ is nonzero if and only if $\left[h^{*} \beta-\beta\right] \neq 0$ on $K$. Moreover, $\Gamma=0$.

\section{2 -forms on the mapping torus}

Let $\Sigma(S, h)=(S \times[0,1]) /(x, 1) \sim(h(x), 0)$ be the mapping torus of $(S, \omega)$. It fibers over the circle with fiber $S$.

There is a natural closed 2-form $\omega_{h}$ on $\Sigma(S, h)$, which is obtained by setting $\omega_{h}=\omega$ on $S \times[0,1]$ and identifying via the symplectomorphism $h$. The 2 -form $\omega_{h}$ pulls back 
to $\omega$ on $S \times\{t\}, t \in[0,1]$, and its kernel is directed by $\partial_{t}$, where $t$ is the coordinate for $[0,1]$.

We have the following lemmas:

Lemma 2.1 Suppose $\omega$ is exact. If $h$ is the first return map of a Reeb vector field $R_{\alpha}$ where $\alpha$ satisfies $\left.d \alpha\right|_{S \times\{0\}}=\omega$, then $h$ is a symplectomorphism of $(S, \omega)$. Moreover, $d \alpha=\omega_{h}$.

Proof Consider the contact 1-form $\alpha=f d t+\beta$ on $S \times[0,1]$, where $f=f(x, t)$ is a function and $\beta=\beta(x, t)$ is a 1 -form in the $S$-direction. Write $\beta_{t}(x)=\beta(x, t)$. We compute

$$
d \alpha=d_{S} f \wedge d t+d_{S} \beta_{t}+d t \wedge \dot{\beta}_{t},
$$

where $d_{S}$ is the exterior derivative in the $S$-direction and $\dot{\beta}_{t}=d \beta_{t} / d t$. By the condition $\left.d \alpha\right|_{S \times\{0\}}=\omega$, we have $d_{S} \beta_{0}=\omega$. Since we can normalize $R_{\alpha}=g \partial_{t}$, where $g=g(x, t)$, it follows that $i_{R_{\alpha}} d \alpha=g\left(-d_{S} f+\dot{\beta}_{t}\right)=0$ and $\dot{\beta}_{t}=d_{S} f$ is an exact form on $S$. Hence $d_{S} \beta_{t}$ is independent of $t$ and equals $\omega$. This shows that $d \alpha=d_{S} \beta_{t}=\omega_{h}$. By the invariance of $\alpha$ under the map $(x, t) \mapsto(h(x), t-1)$, we see that $h$ preserves $\omega$.

Lemma 2.2 Suppose $\omega$ is exact and the flux of $h$ is nonzero. Then $\left[\omega_{h}\right]$ is nonzero in $H^{2}(\Sigma(S, h) ; \mathbb{Z})$. Hence $h$ cannot be realized as the first return map of a Reeb vector field $R_{\alpha}$.

Proof Let $\gamma$ be a curve in $S$ such that $F_{h}(\gamma)$ is nonzero. Then $-\gamma$ and $h(\gamma)$ bound a subsurface $C \subset S \times\{0\}$ so that $\int_{C} \omega \neq 0$. We construct a closed 2-cycle $C^{\prime}$ in $\Sigma(S, h)$ by gluing $\gamma \times[0,1]$ with $C$. Now we see that $\int_{C^{\prime}} \omega_{h}=\int_{C} \omega \neq 0$. Hence $\left[\omega_{h}\right] \neq 0$ in $H^{2}(\Sigma(S, h) ; \mathbb{Z})$. By Lemma $2.1, h$ cannot be the first return map of a Reeb vector field.

Conversely, when $\omega$ is exact and $h$ is the identity near $\partial S$, we have a criterion, due to Giroux (see Colin [2]), to realize $h$ as the first return map of a Reeb vector field. The condition that $h=$ id near $\partial S$ is not realized in general for pseudo-Anosov homeomorphisms, but in practice it is possible to deform the diffeomorphism near $\partial S$ so that it is the identity, without altering the sets of periodic points too much; see Colin and Honda [3].

Lemma 2.3 (Giroux) Let $(S, \omega=d \beta)$ be a compact exact symplectic manifold and $h$ be a symplectomorphism of $(S, \omega)$, which is the identity near $\partial S$. If $\left[h^{*} \beta-\beta\right]=0$ in $H^{1}(S ; \mathbb{R})$, then there exists a contact form $\alpha$ on $\Sigma(S, h)$ and a Reeb vector field $R_{\alpha}$ whose first return map on one fiber is $h$. 
Proof We have that $h^{*} \beta-\beta=d f$. Note that $d f=0$ near $\partial S$ since $h=$ id near $\partial S$. One can always translate $f$ so that $f$ is strictly positive on $S$ and is constant near $\partial S$. The 1 -form $\alpha=d t+\beta$ is a contact form on $S \times \mathbb{R}$ whose Reeb vector field is $\partial_{t}$. It is invariant under the diffeomorphism

$$
H:(x, t) \mapsto(h(x), t-f(x)),
$$

and thus induces a contact form $\alpha$ on $\Sigma(S, h) \simeq(S \times \mathbb{R}) /((x, t) \sim H(x, t))$.

\subsection{Surface case}

Let us now specialize to the case of interest: $S$ is a compact oriented surface, $\omega$ is an area form on $S$ and $h$ is an area-preserving diffeomorphism of $(S, \omega)$. Let us assume without loss of generality that the $\omega$-area of $S$ is 1 . If $\partial S=\varnothing$, then $\Gamma=\mathbb{Z}$ since $H_{2}(S ; \mathbb{Z})$ is generated by $[S]$. On the other hand, if $\partial S \neq \varnothing$, then $\Gamma=0$ and the exactness of $\omega$ is automatically satisfied.

The goal of this note is to prove the following:

Theorem 2.4 There exist a compact surface $S$ with empty (resp. nonempty) boundary and a pseudo-Anosov homeomorphism $h$ of $S$ with $h_{*}=\mathrm{id}$, whose flux with respect to the singular $h$-invariant area form $\omega$ is nonzero, as viewed in $\mathbb{R} / \mathbb{Z}$ (resp. $\mathbb{R}$ ).

We now discuss a technical issue, namely the fact that $h$ is only $C^{0}$ at the singular set $L=\left\{p_{1}, \ldots, p_{k}\right\}$ of the stable/unstable foliations. Let $\omega$ be the $h$-invariant singular area form given by the product $\mu^{u} \otimes \mu^{s}$ of both transverse measures. It is singular in the sense that it is a 2 -form which vanishes on $L$. As a measure it is equivalent to any Lebesgue measure on $S$. Hence, according to a theorem of Oxtoby and Ulam [7], it is conjugated by a homeomorphism to a smooth area form.

Instead of the Oxtoby-Ulam approach, our approach will be based on Moser's lemma. Let $D$ be an arbitrarily small open neighborhood of $L$ so that each connected component of $D$ is a polygonal region whose boundary consists of subarcs of leaves of $\mathcal{F}^{s}$ or $\mathcal{F}^{u}$. Then we have the following:

Lemma 2.5 There exist an everywhere smooth area form $\omega^{\prime}$ on $S$ and a diffeomorphism $h^{\prime}$, which coincide respectively with $\omega$ and $h$ outside of $D$ and satisfy (i) $\int_{D_{0}} \omega=\int_{D_{0}} \omega^{\prime}$ for each connected component $D_{0}$ of $D$ and (ii) $\left(h^{\prime}\right)^{*} \omega^{\prime}=\omega^{\prime}$.

Proof Let $\omega^{\prime}$ be an area form which coincides with $\omega$ on $S-(D \cap h(D))$, and has the same area as $\omega$ on each connected component $D_{0}$ of $D$. (By using an 
auxiliary area form on $S$, the construction of such an $\omega^{\prime}$ becomes equivalent to the extension of a positive smooth function with a fixed integral.) There also exists a smooth diffeomorphism $\psi$ of $S$ which coincides with $h$ on $S-D$. Note that the germ of $h$ along $\partial D$ extends to an embedding of $D$ into $S$. By the construction of $\omega^{\prime}$ and $\psi$, we have $\psi^{*} \omega^{\prime}=\omega^{\prime}$ on $S-D$.

We now claim that

$$
\int_{D_{0}} \psi^{*} \omega^{\prime}=\int_{D_{0}} \omega^{\prime}
$$

for each component $D_{0}$ of $D$. We have

$$
\int_{D_{0}} \omega^{\prime}=\int_{D_{0}} \omega=\int_{D_{0}} h^{*} \omega=\int_{h\left(D_{0}\right)} \omega
$$

by our choice of $\omega^{\prime}$ and the $h$-invariance of $\omega$. On the other hand, we have

$$
\int_{D_{0}} \psi^{*} \omega^{\prime}=\int_{h\left(D_{0}\right)} \omega^{\prime}
$$

by a change of variables. If $D_{0}^{\prime}$ is the component of $D$ that nontrivially intersects $h\left(D_{0}\right)$, then

$$
\int_{D_{0}^{\prime} \cap h\left(D_{0}\right)} \omega=\int_{D_{0}^{\prime} \cap h\left(D_{0}\right)} \omega^{\prime},
$$

since

$$
\int_{D_{0}^{\prime}} \omega=\int_{D_{0}^{\prime}} \omega^{\prime}
$$

and $\omega=\omega^{\prime}$ on $D_{0}^{\prime}-h\left(D_{0}\right)$. From this we deduce that

$$
\int_{h\left(D_{0}\right)} \omega=\int_{h\left(D_{0}\right)} \omega^{\prime}
$$

The claimed equality follows.

Finally, Moser's lemma applies on $D$ to the pair of area forms $\omega^{\prime}$ and $\psi^{*} \omega^{\prime}$. It yields a diffeomorphism $\varphi$ of $D$ which is the identity near the boundary (hence extends to $S$ by the identity of $\left.S^{\prime}\right)$ such that $\varphi^{*}\left(\psi^{*} \omega^{\prime}\right)=\omega^{\prime}$. We set $h^{\prime}=\psi \circ \varphi$. This diffeomorphism meets the required condition both on $S^{\prime}$ and $D$, hence on $S$.

If we choose $\gamma$ so that both $\gamma$ and $h^{\prime}(\gamma)$ avoid the small neighborhood $D$ of the singular locus $L$ (after isotopy), then we see that $F_{h}(\gamma)=F_{h^{\prime}}(\gamma)$. Since the flux only depends on the curve up to isotopy, it follows that $F_{h}=F_{h^{\prime}}$. 
Remark When $\mathcal{F}^{s}$ and $\mathcal{F}^{u}$ are orientable, the transverse measures define 1-forms that are closed but not exact. They are eigenvectors for $h^{*}$ with eigenvalues $\lambda$ and $1 / \lambda$. Thus if $h_{*}=\mathrm{id}$, then the foliations are not orientable.

\section{Proof of Theorem 2.4}

Let $S=S_{g}$ be a closed oriented surface of genus $g$ and $\alpha$ and $\beta$ be two 1-dimensional submanifolds of $S$, ie, the union of disjoint simple closed curves.

We recall that $\alpha$ and $\beta$ fill $S$ if $\alpha$ and $\beta$ intersect transversely and minimally and if each region of $S-(\alpha \cup \beta)$ is a $2 n$-gon with $n>1$. Such a system of curves allows one to define two systems of flat charts, the $\alpha$ - and the $\beta$-charts, in the following way: The set $\alpha \cup \beta$ gives a cell decomposition of $S$. Consider its dual cell decomposition. (By this we mean we place a vertex $v_{P_{i}}$ in the interior of each component $P_{i}$ of $S-(\alpha \cup \beta)$. If $P_{i}$ and $P_{j}$ share an edge of $\alpha \cup \beta$, then take an edge from $v_{P_{i}}$ to $v_{P_{j}}$ which passes through the common edge of $\alpha \cup \beta$ exactly once.) Let $E_{\beta}$ be the union of edges of the dual cellular decomposition that meet $\beta$. Then $E_{\beta}$ cuts $S$ into annuli whose cores are the components of $\alpha$ that we call the $\alpha$-charts. The $\beta$-charts are defined similarly.

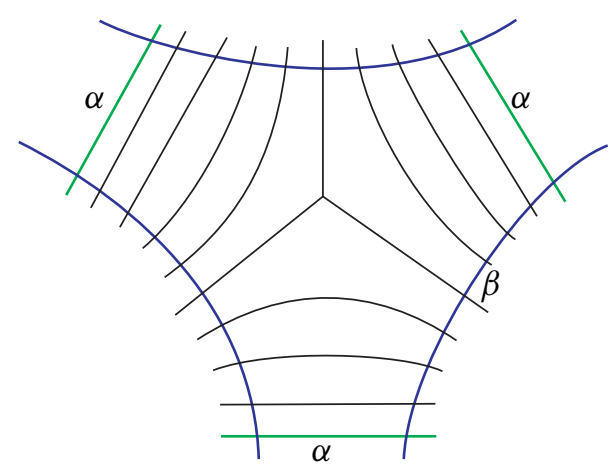

Figure 1: Dual cell decomposition meeting the $\beta$ curves, together with flat geodesics parallel to $\alpha$

Note that there is one chart for each curve and hence each chart can be viewed as a thickening of an appropriate $\alpha$ - or $\beta$-curve. These charts are equipped with a singular flat metric $g$ which is standard on each little square, corresponding to intersections of $\alpha$ - and $\beta$-charts, as explained in [5, Exposé 13, Section III]. (In particular, the $\alpha$-metric and the $\beta$-metric coincide on the squares.)

We will construct our example on a surface $S_{5}$ of genus 5 . 
Lemma 3.1 There exist two multicurves $\alpha=\alpha_{1} \cup \alpha_{2}$ and $\beta=\beta_{1} \cup \beta_{2}$ on $S_{5}$ where:

- $\alpha$ and $\beta$ fill $S_{5}$;

- $\alpha_{1}$ and $\beta_{1}$ are disjoint and form a bounding pair;

- $\alpha_{2}$ and $\beta_{2}$ are separating curves;

- $\#\left(\alpha_{1} \cap \beta_{2}\right)=\#\left(\alpha_{2} \cap \beta_{1}\right)=2$;

- $\#\left(\alpha_{2} \cap \beta_{2}\right)=16$.

Proof We start with a genus 2 surface $S_{2}^{\prime}$, together with simple closed curves $\alpha_{2}^{\prime}$ and $\beta_{2}^{\prime}$ which are both nullhomologous in $S_{2}^{\prime}$, fill $S_{2}^{\prime}$ and intersect 8 times. See Figure 2. (To see that $\beta_{2}^{\prime}$ separates, take the algebraic intersection number with a suitable basis for $H_{2}\left(S_{2}^{\prime} ; \mathbb{Z}\right)$.) Now, two regions $H_{1}^{\prime}$ and $H_{2}^{\prime}$ of $S_{2}^{\prime} \backslash\left(\alpha_{2}^{\prime} \cup \beta_{2}^{\prime}\right)$ are 8 -gons. For $i=1,2$, pick a disk $D_{i}^{\prime} \subset \operatorname{Int}\left(H_{i}^{\prime}\right)$.

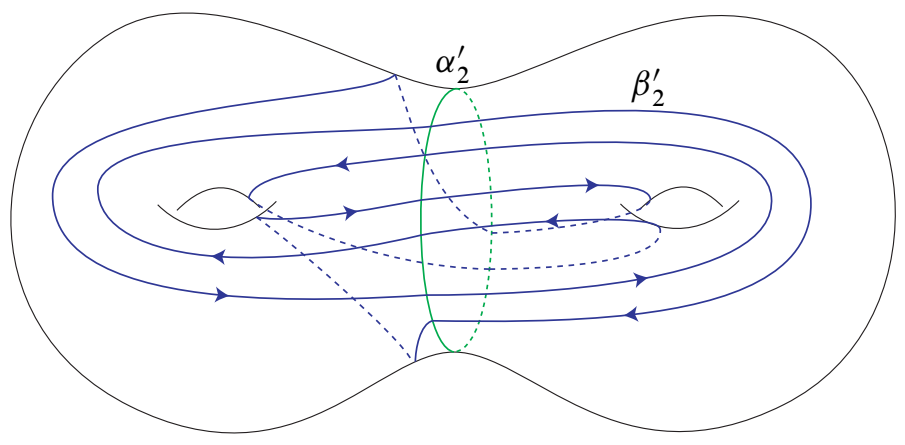

Figure 2: The genus 2 surface $S_{2}^{\prime}$

We now take a second copy $\left(S_{2}^{\prime \prime}, \alpha_{2}^{\prime \prime}, \beta_{2}^{\prime \prime}, D_{1}^{\prime \prime}, D_{2}^{\prime \prime}\right)$ of $\left(S_{2}^{\prime}, \alpha_{2}^{\prime}, \beta_{2}^{\prime}, D_{1}^{\prime}, D_{2}^{\prime}\right)$ and glue $S_{2}^{\prime} \backslash\left(D_{1}^{\prime} \cup D_{2}^{\prime}\right)$ to $S_{2}^{\prime \prime} \backslash\left(D_{1}^{\prime \prime} \cup D_{2}^{\prime \prime}\right)$ by identifying $\partial D_{i}^{\prime}$ and $\partial D_{i}^{\prime \prime}, i=1,2$. We call $S_{5}$ the resulting surface. See Figure 3. Let $\alpha_{1}=\partial D_{1}^{\prime}=\partial D_{1}^{\prime \prime}$ and $\beta_{1}=\partial D_{2}^{\prime}=\partial D_{2}^{\prime \prime}$.

Next, take one connected component of $\beta_{2}^{\prime} \cap \partial H_{1}^{\prime}$ and one connected component of $\beta_{2}^{\prime \prime} \cap \partial H_{1}^{\prime \prime}$, and make the connected sum of these two components along an arc which crosses $\alpha_{1}$ exactly once and stays inside $\left(H_{1}^{\prime} \backslash \operatorname{Int}\left(D_{1}^{\prime}\right)\right) \cup\left(H_{1}^{\prime \prime} \backslash \operatorname{Int}\left(D_{1}^{\prime \prime}\right)\right)$. We call $\beta_{2}$ the result of this sum of $\beta_{2}^{\prime}$ and $\beta_{2}^{\prime \prime}$. By construction, $\#\left(\alpha_{1} \cap \beta_{2}\right)=2$. Now do the same operation with components of $\alpha_{2}^{\prime}$ and $\alpha_{2}^{\prime \prime}$ in $\partial H_{2}^{\prime}$ and $\partial H_{2}^{\prime \prime}$, so that the resulting curve $\alpha_{2}$ satisfies $\#\left(\alpha_{2} \cap \beta_{1}\right)=2$.

By construction, we see that $\#\left(\alpha_{2} \cap \beta_{2}\right)=8+8=16$. The families $\alpha=\alpha_{1} \cup \alpha_{2}$ and $\beta=\beta_{1} \cup \beta_{2}$ fill $S_{5}$. Since $\alpha_{2}^{\prime}$ and $\alpha_{2}^{\prime \prime}$ were nullhomologous, the same also holds for $\alpha_{2}$. Finally it is clear that $\alpha_{1}$ and $\beta_{1}$ are disjoint and cobordant in $S_{5}$. 


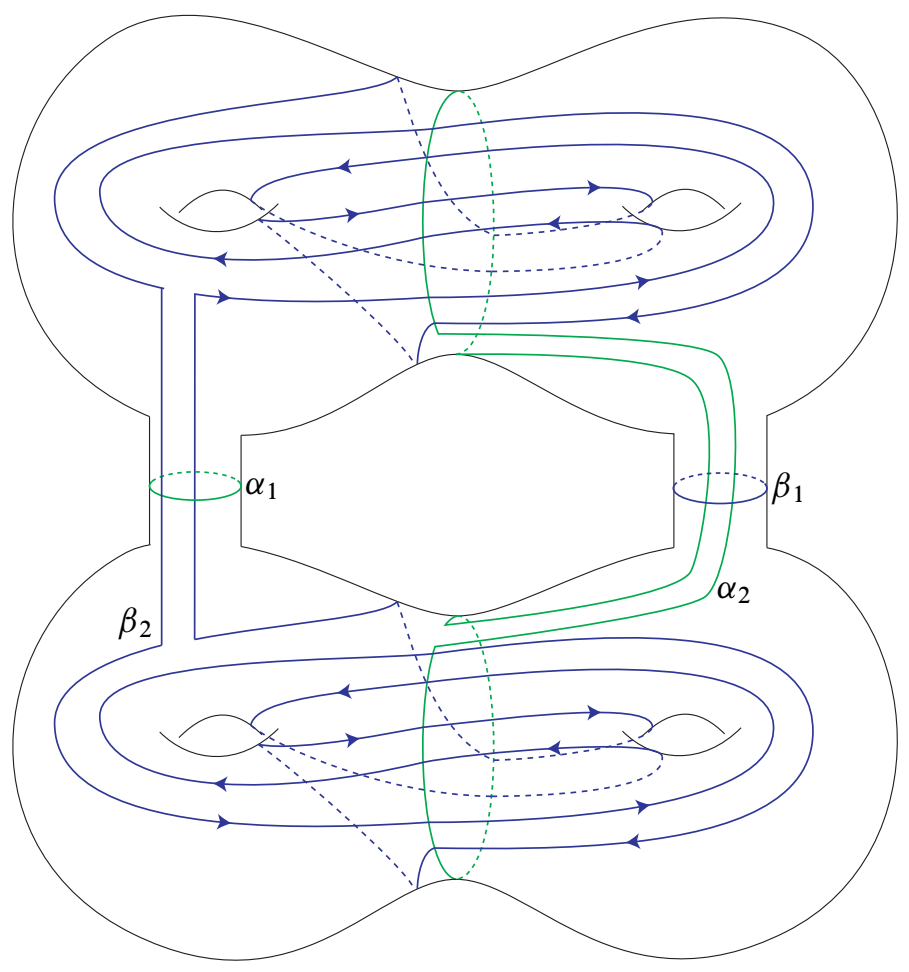

Figure 3: The genus 5 surface $S_{5}$

The system $\alpha=\alpha_{1} \cup \alpha_{2}$, provided by Lemma 3.1, comes with two (oriented) $\alpha$-charts $U_{1} \supset \alpha_{1}$ and $U_{2} \supset \alpha_{2}$, where $U_{i}=\left[0, n_{i}\right] \times[0,1] /(0, y) \sim\left(n_{i}, y\right), n_{i}$ denotes $\#\left(\alpha_{i} \cap \beta\right)$, namely $n_{1}=2, n_{2}=18$ and $\alpha_{i}=\left[0, n_{i}\right] \times\{1 / 2\} / \sim$. Similarly, there are two $\beta$-charts $V_{1}$ and $V_{2}$, of the form $[0,1] \times\left[0, m_{i}\right] /(x, 0) \sim\left(x, m_{i}\right)$, where $m_{i}=\#\left(\beta_{i} \cap \alpha\right)$, ie, $m_{1}=2, m_{2}=18$, and $\beta_{i}=\{1 / 2\} \times\left[0, m_{i}\right] / \sim$. In what follows, we equip $S_{5}$ with the flat metric associated to the system $\alpha$ and $\beta$ and compute areas using this metric, normalized so that the total area of $S$ is 1 .

We will denote $\left[\tau_{\eta}\right]$ the mapping class of a positive Dehn twist about the closed curve $\eta$. The class $\left[\tau_{\alpha_{i}}\right]$ admits an affine representative $\tau_{\alpha_{i}}$ which is given on $U_{i}$ by the matrix

$$
\left(\begin{array}{cc}
1 & n_{i} \\
0 & 1
\end{array}\right)
$$

and is the identity on $U_{j}$ for $j \neq i$. 
Similarly, $\left[\tau_{\beta_{i}}^{-1}\right]$ admits an affine representative $\tau_{\beta_{i}}^{-1}$ which is given on $V_{i}$ by the matrix

$$
\left(\begin{array}{cc}
1 & 0 \\
m_{i} & 1
\end{array}\right)
$$

and is the identity on $V_{j}$ for $j \neq i$.

Lemma 3.2 The map $h=\tau_{\alpha_{2}} \circ \tau_{\alpha_{1}}^{9} \circ \tau_{\beta_{1}}^{-9} \circ \tau_{\beta_{2}}^{-1}$ is a pseudo-Anosov homeomorphism which acts by the identity on $H_{1}(S ; \mathbb{Z})$.

Proof On both $U_{1}$ and $U_{2}$, the composition $\tau_{\alpha_{2}} \circ \tau_{\alpha_{1}}^{9}$ is given by the matrix

$$
\left(\begin{array}{cc}
1 & 18 \\
0 & 1
\end{array}\right)
$$

and thus is a smooth representative of its mapping class outside the singular points of the flat structure. Similarly, on both $V_{1}$ and $V_{2}$, the composition $\tau_{\beta_{1}}^{-9} \circ \tau_{\beta_{2}}^{-1}$ is given by the matrix

$$
\left(\begin{array}{cc}
1 & 0 \\
18 & 1
\end{array}\right)
$$

As a result, the homeomorphism $h$ is given away from the singular points of the flat structure by the matrix

$$
\left(\begin{array}{cc}
325 & 18 \\
18 & 1
\end{array}\right)
$$

Since the trace of the matrix is $>2, h$ is pseudo-Anosov. It preserves the area coming from the singular flat metric on the charts.

Since $\alpha_{2}$ and $\beta_{2}$ are homologous to zero and $\alpha_{1}$ and $\beta_{1}$ form a bounding pair, $h$ induces the identity on homology.

Lemma 3.3 The flux of $h$ is nonzero, when viewed in $\mathbb{R} / \mathbb{Z}$. More precisely, if $\gamma$ is a curve so that $\tau_{\beta_{2}}^{-1}(\gamma)$ has geometric intersection one with each of $\alpha_{1}$ and $\beta_{1}$, then $F_{h}([\gamma]) \neq 0$.

Proof Let $\delta$ be a closed geodesic with respect to the singular flat metric which corresponds to the singular flat coordinate system. In other words, $\delta$ is a piecewise affine curve, with corners at singularities of the affine structure.

First we claim that $\tau_{\beta_{2}}^{-1}$ has zero flux, ie, the area between $\delta$ and $\tau_{\beta_{2}}^{-1}(\delta)$ is zero for all $\delta$. (Note that $\tau_{\beta_{2}}^{-1}(\delta)$ is not necessarily a flat geodesic even if $\delta$ is.) Indeed, since $\tau_{\beta_{2}}^{-1}$ is the identity on $V_{1}$, we only have to look on $V_{2}$. The curve $\delta$ intersects $V_{2}$ along a finite union of affine $\operatorname{arcs} a_{1}, \ldots, a_{n}$. For any such $a_{i}$, the concatenation $\bar{a}_{i}$ of $-a_{i}$ 
and $\tau_{\beta_{2}}^{-1}\left(a_{i}\right)$ divides $V_{2}$ into two components with the same area. This means that the area between $\bar{a}_{i}$ and $\beta_{2}=\{1 / 2\} \times[0,18] / \sim$ is zero. Thus, $\bar{a}_{i}$ bounds a subsurface in $S$ with the same area as the surface bounded by $\beta_{2}=\{1 / 2\} \times[0,18] / \sim$. The sign of this area depends on the sign of the intersection of $a_{i}$ with $\beta_{2}$. Now observe that $\tau_{\beta_{2}}^{-1}(\delta)-\delta=\bigcup_{1 \leq i \leq n} \bar{a}_{i}$. Since $\beta_{2}$ is homologous to zero, it has as many positive intersections with $\delta$ as negative intersections. Thus the total signed area between $\delta$ and $\tau_{\beta_{2}}^{-1}(\delta)$ is the total signed area bounded by $\bigcup_{1 \leq i \leq n} \bar{a}_{i}$, which in turn is zero. Similarly, we see that $\tau_{\alpha_{2}}$ has zero flux.

Next suppose the geodesic $\delta$ has geometric intersection one with each of $\alpha_{1}$ and $\beta_{1}$. We claim that the area between $\delta$ and $\tau_{\alpha_{1}} \circ \tau_{\beta_{1}}^{-1}(\delta)$ equals the area $A$ bounded by $\alpha_{1} \cup \beta_{1}$ in $S$. Since $\alpha_{1} \cap \beta_{1}=\varnothing$, it follows that $\operatorname{Int}\left(U_{1}\right) \cap \operatorname{Int}\left(V_{1}\right)=\varnothing$ and the affine representatives $\tau_{\alpha_{1}}$ and $\tau_{\beta_{1}}^{-1}$ commute. The curve $\delta$ intersects $V_{1}$ along a connected affine arc $b$ and $U_{1}$ along a connected affine arc $a$. The concatenation of $-b$ and $\tau_{\beta_{1}}^{-1}(b)$ is a closed curve $\bar{b}$ which cuts $V_{1}$ into two components of the same area. Similarly, $\bar{a}$, obtained as the concatenation of $-a$ and $\tau_{\alpha_{1}}(a)$, divides $U_{1}$ into two components of the same area. Then $\tau_{\alpha_{1}} \circ \tau_{\beta_{1}}^{-1}(\delta)-\delta$ equals $\bar{a} \cup \bar{b}$, and $\delta$ and $\tau_{\alpha_{1}} \circ \tau_{\beta_{1}}^{-1}(\delta)$ cobound a subsurface in $S_{5}$ of area $A$. By the commutativity of $\tau_{\alpha_{1}}$ and $\tau_{\beta_{1}}^{-1}$, we have $\tau_{\alpha_{1}}^{9} \circ \tau_{\beta_{1}}^{-9}=\left(\tau_{\alpha_{1}} \circ \tau_{\beta_{1}}^{-1}\right)^{9}$. Hence the area between $\delta$ and $\tau_{\alpha_{1}}^{9} \circ \tau_{\beta_{1}}^{-9}(\delta)$ is $9 A$.

We now claim that $A=(1 / 2) \operatorname{Area}(S)=1 / 2$. This is due to the symmetry of the $\alpha-$ and $\beta$-charts: The chart $U_{1}$ is decomposed by $\alpha_{1}=[0,2] \times\{1 / 2\} / \sim$ into two pieces with the same area. On the other hand, $U_{2}$ is decomposed by $\beta_{1}$ into two rectangles $R_{1}$ and $R_{2}$. On each $R_{i}$, the number of intersections between $\alpha_{2}$ and $\beta_{2}$ is 8 . Hence $\operatorname{Area}\left(R_{1}\right)=\operatorname{Area}\left(R_{2}\right)$. We conclude that the area between $\delta$ and $\tau_{\alpha_{1}}^{9} \circ \tau_{\beta_{1}}^{-9}(\delta)$ is $9 A=9 / 2 \equiv 1 / 2 \in \mathbb{R} / \mathbb{Z}$.

By putting together the above calculations and observing that $F_{h}([\gamma])$ only depends on the isotopy class of $\gamma$, we see that $F_{h}([\gamma])=A=1 / 2$.

This completes the proof of Theorem 2.4 when $\partial S=\varnothing$.

To treat the case with boundary, we notice that the homeomorphism we have constructed fixes the singular points of the invariant foliations. We pick one of them and blow up the surface at this point. The homeomorphism $h$ lifts to a pseudo-Anosov homeomorphism on the blown-up surface $\bar{S}$ which fixes the blown-up foliations. It also induces $\mathrm{id}_{*}$ on $H_{1}(\bar{S} ; \mathbb{Z})$ and has nonzero flux.

On the other hand, it is easy to construct pseudo-Anosov homeomorphisms with vanishing flux that act trivially on $H_{1}(S ; \mathbb{Z})$. Let $\alpha_{1}, \beta_{1}$ be simple closed curves which fill $S$ and are both nullhomologous. As explained in [5, Exposé 13, Section III], if we compose twists along these curves (positive Dehn twists along $\alpha_{1}$ represented 
by $\tau_{\alpha_{1}}$ and negative Dehn twists along $\beta_{1}$ represented by $\tau_{\beta_{1}}^{-1}$, where we use at least one $\tau_{\alpha_{1}}$ and at least one $\tau_{\beta_{1}}^{-1}$ ), we obtain a pseudo-Anosov homeomorphism. The argument developed in the proof of Lemma 3.3 tells us that the flux is always zero. More precisely, consider the singular flat metric compatible with $\alpha_{1}$ and $\beta_{1}$, and let $\delta$ be a closed curve represented by a flat geodesic. As in the second paragraph of the proof of Lemma 3.3, the area between $\delta$ and $\tau_{\alpha_{1}}(\delta)$ is zero for all $\delta$, since $\alpha_{1}$ is separating. Similarly, the area between $\delta$ and $\tau_{\beta_{1}}^{-1}(\delta)$ is zero for all $\delta$. Hence, if $h$ is any composition of $\tau_{\alpha_{1}}$ and $\tau_{\beta_{1}}^{-1}$ (both with zero flux), then it also has zero flux. This justifies the fact that, in the proof of Theorem 2.4, we have to look at more elaborate examples to find nonzero flux.

Another case when $h$ can be realized as the first return map of a Reeb flow is when $h^{*}-$ id is invertible and $\partial S \neq \varnothing$. We learned the following lemma from Yasha Eliashberg.

Lemma 3.4 Let $h$ be a diffeomorphism of a surface $S$ with nonempty boundary which preserves an area form $\omega$. If 1 is not an eigenvalue of $h^{*}$, then $\omega$ admits a primitive $\beta$ such that $\left[h^{*} \beta-\beta\right]=0$ in $H^{1}(S ; \mathbb{R})$.

Proof Pick any primitive $\beta_{0}$ of $\omega$. By hypothesis, the map $h^{*}-\mathrm{id}$ is surjective. Thus, one can find $[\theta] \in H^{1}(S ; \mathbb{R})$ such that $\left[h^{*} \beta_{0}-\beta_{0}\right]=\left(h^{*}-\mathrm{id}\right)[\theta]$. Now we have that $\beta=\beta_{0}-\theta$ is a primitive of $\omega$ and that $\left[h^{*} \beta-\beta\right]=0$ in $H^{1}(S ; \mathbb{R})$.

Now, by applying Lemma 2.3, $h$ can be realized as the first return map of a Reeb vector field.

We end this section with the following questions:

Question 3.5 Is it possible to find a pseudo-Anosov homeomorphism of a surface $S$ which acts trivially on $H_{1}(S ; \mathbb{Z})$ and takes some noncontractible curve $\gamma$ to a curve $h(\gamma)$ that can be isotoped away from $\gamma ?^{1}$

If yes, the flux of such a pseudo-Anosov homeomorphism would automatically be nonzero.

Question 3.6 Let $g$ and $h$ be two pseudo-Anosov homeomorphisms acting trivially on $H_{1}(S ; \mathbb{Z})$ such that the composition $g \circ h$ is isotopic to a pseudo-Anosov homeomorphism $f$. Suppose the flux of $g$ is zero and the flux of $h$ is nonzero. Is the flux of $f$ nonzero?

If yes, this procedure would allow us to produce many pseudo-Anosov homeomorphisms with nonzero flux.

\footnotetext{
${ }^{1}$ Dan Margalit has informed us of an example of a pseudo-Anosov homeomorphism on a genus 3 surface with this property. His example would therefore also have nonzero flux.
} 


\section{A question}

There is an invariant of an isotopy class of surface diffeomorphisms $[h]$ which is defined in a manner much like the flux. We thank Ian Agol for bringing this to the authors' attention. Let $S$ be a hyperbolic surface with geodesic boundary. If $[\gamma] \in K$, ie, $[h(\gamma)-\gamma]=0$, then represent $h(\gamma)$ and $\gamma$ by geodesics, and compute the area bounded by the two geodesics. By the Gauss-Bonnet theorem, this area equals $-2 \pi \chi(A)$, where $A$ is a surface between the two geodesics. Here the Euler characteristic $\chi(A)$ is more precisely an Euler measure, ie, it is computed with signs: if $-A$ denotes $A$ with reversed orientation, then one has $\chi(-A)=-\chi(A)$. This gives rise to a map

$$
G_{[h]}: K \rightarrow \mathbb{R} / \Gamma,
$$

where $\Gamma=2 \pi \chi(S) \mathbb{Z}$ when $S$ is closed and $\Gamma=0$ when $S$ has boundary. When restricted to the Torelli group $\mathcal{T}(S)$, we have a homomorphism:

$$
\begin{aligned}
G: \mathcal{T}(S) & \rightarrow H^{1}(S ; \mathbb{R} / \Gamma) \simeq \operatorname{Hom}\left(H_{1}(S ; \mathbb{Z}), \mathbb{R} / \Gamma\right), \\
{[h] } & \mapsto G_{[h]} .
\end{aligned}
$$

Since the pseudo-Anosov representative of a mapping class is basically unique, we ask:

Question 4.1 Is $F_{h}=G_{[h]}$ for $h$ pseudo-Anosov and in $\mathcal{T}(S)$, up to an overall constant factor?

Finally, we briefly discuss the relationship to the monotonicity condition for an areapreserving diffeomorphism $h$, described by Seidel [8]. Suppose that $\chi(S)<0$. On $\Sigma(S, h)$ consider the tangent bundle $W$ to the fibers and let $c_{1}(W)$ be its first Chern class. The monotonicity condition requires that $\left[\omega_{h}\right]=\lambda c_{1}(W)$ for some real number $\lambda$. Using the notation from Lemma 2.2, one can verify that $\left\langle c_{1}(W), C^{\prime}\right\rangle=\chi(C)$ for homology classes of type $C^{\prime}$. Here $C$ is the surface with $\partial C=h(\gamma)-\gamma$. This means that monotonicity holds if and only if $F_{h}$ and $G_{[h]}$ are proportional. (A similar, but slightly more complicated, monotonicity condition also appears in the definition of periodic Floer homology of $h$. See Hutchings and Sullivan [6].)

Acknowledgements We thank Yasha Eliashberg and Sylvain Gervais for very helpful conversations. We also thank Andrew Cotton-Clay and Dan Margalit for their comments on the first version of the paper.

VC was supported by the Institut Universitaire de France and the ANR Symplexe. KH was supported by an NSF CAREER Award (DMS-0237386) and NSF Grant DMS-0805352. 


\section{References}

[1] E Calabi, On the group of automorphisms of a symplectic manifold, from: "Problems in analysis (Lectures at the Sympos. in honor of Salomon Bochner, Princeton Univ., 1969)”, Princeton Univ. Press (1970) 1-26 MR0350776

[2] V Colin, Livres ouverts en géométrie de contact (d'après Emmanuel Giroux), Séminaire Bourbaki, Exposé 969, Astérisque 311, Soc. Math. France, Paris (2008)

[3] V Colin, K Honda, Reeb vector fields and open book decompositions arXiv: 0809.5088

[4] V Colin, K Honda, Stabilizing the monodromy of an open book decomposition, Geom. Dedicata 132 (2008) 95-103 MR2396912

[5] A Fathi, F Laudenbach, V Poénaru, Travaux de Thurston sur les surfaces, Soc. Math. France, Paris (1991) MR1134426 Reprint of Astérisque 66-67 MR0568308

[6] M Hutchings, M Sullivan, The periodic Floer homology of a Dehn twist, Algebr. Geom. Topol. 5 (2005) 301-354 MR2135555

[7] J C Oxtoby, S M Ulam, Measure-preserving homeomorphisms and metrical transitivity, Ann. of Math. (2) 42 (1941) 874-920 MR0005803

[8] P Seidel, Symplectic Floer homology and the mapping class group, Pacific J. Math. 206 (2002) 219-229 MR1924827

VC, FL: Université de Nantes UMR 6629 du CNRS

Laboratoire de mathématiques Jean Leray, 2 rue de la Houssinière, Nantes, 44322, France KH: University of Southern California

Department of Mathematics, 3620 S Vermont Ave, Los Angeles, California 90089, USA vincent.colin@univ-nantes.fr, khonda@usc.edu, francois. laudenbach@univ-nantes.fr

http://www. math.sciences. univ-nantes.fr/ vcolin/, http://rcf.usc.edu/ khonda

Received: 8 September 2008 